\title{
ACTIVE MASK SEGMENTATION FOR THE CELL-VOLUME COMPUTATION AND GOLGI-BODY SEGMENTATION OF HELA CELL IMAGES
}

\author{
Gowri Srinivasa ${ }^{1}$, Matthew Fickus $^{4}$, Manuel N. Gonzalez-Rivero ${ }^{2}$, Sarah Yichia Hsieh ${ }^{1,2}$, \\ Yusong Guo ${ }^{3}$, Adam D. Linstedt ${ }^{3}$ and Jelena Kovačević ${ }^{1,2}$ \\ ${ }^{1}$ Dept. of Biomedical Engineering and Center for Bioimage Informatics \\ ${ }^{2}$ Dept. of Electrical and Computer Engineering \\ ${ }^{3}$ Dept. of Biological Sciences, Carnegie Mellon University, Pittsburgh, USA \\ ${ }^{4}$ Dept. of Mathematics and Statistics, Air Force Institute of Technology, Wright-Patterson AFB, USA
}

\begin{abstract}
We present a novel active mask framework for the segmentation of fluorescence microscope images of cells, and in particular, for the segmentation of the Golgi body as well as cellvolume computation. We demonstrate that the algorithm is able to efficiently segment a stack of images and successfully assign multiple pieces of the Golgi body in a 2D image to the cell to which they belong. Further, we demonstrate that our algorithm is more accurate than manual segmentation of these images.
\end{abstract} tion

Index Terms - active contours, active mask, segmenta-

\section{INTRODUCTION}

The Golgi body is an organelle in eukaryotic cells that mediates the processing and sorting of proteins and lipids in the final stages of their biosynthesis. It is a dynamic organelle that undergoes rapid cycles of assembly and disassembly in response to cell division and differentiation. The cytoplasmic surface of the Golgi body is a site for numerous important signaling pathways [1], and defects in the components of these pathways are responsible for many human diseases [2]. As the understanding of the molecular basis of these defects is paving the way for new therapeutics [3], understanding the Golgi apparatus is of particular interest and is the focus of research of Linstedt et. al. [4, 5].

Biological problem. The current hypothesis being tested by Linstedt is that interaction affinity between vesicle coat complexes and SNARE molecules (and other Golgi proteins) establishes and controls the size of Golgi compartments [4]. These tests depend on a quantitative ratiometric assay comparing Golgi size to its cell size. To date, the experimental tests of this hypothesis resulted in the discovery that COPII

This work was supported in part by NSF through awards ITR-EF0331657 and DMS-0405376 as well as the PA State Tobacco Settlement, Kamlet-Smith Bioinformatics Grant. assembly is regulated by the availability of Golgi proteins in the ER due to a direct interaction between components in the Golgi protein cytoplasmic domains and the COPII component Sar1p [5].

The need for automated segmentation. The assay is fluorescence microscope-based and accurate segmentation of the image is the critical step in determining cell volume. As a segmentation algorithm suited for this application is not available, cell boundaries were segmented manually in each of as many as 20 optical slices. This manual processing is not only time-consuming and error prone but also limits the researchers to only what is discernable to the human eye, necessitating the use of an automated segmentation algorithm.

\section{ACTIVE CONTOUR SEGMENTATION OF FLUORESCENCE MICROSCOPE CELL IMAGES}

Active contour algorithms are today's state-of-the-art in the segmentation community and involve the calculus of variations and partial differential equations. This class of algorithms attempts to find smooth, closed contours that envelop shapes within the image. Here, the contour is comparable to an elastic string that moves according to two kind of forces: internal and external. Internal forces are determined from the intrinsic geometric properties of the contour, such as its curvature. External forces are derived from the image itself, using edge detection or image statistics, for example. While originally modeled as parameterized curves, active contours have more recently been formulated as level sets of a surface [6]. Active contours have only begun to be used to segment biological images [7, 8, 9, 10,11, 12].

Previously, we designed a topology preserving stochastic active contour scheme (TPSTACS) for the segmentation of fluorescence microscope images of cells [10]. This design incorporated concepts from digital topology into the level set formulation of the active contour method [13]. We demonstrated that TPSTACS outperforms the seeded watershed algorithm (widely used in fluorescence microscopy) both by vi- 
sual inspection and quantitative measures of performance.

The success of TPSTACS led to further enhancements such as the introduction of a multiresolution component to the system to reduce the run time of the algorithm [11]. Subsequently, we studied the limitations of the level set formulation of the active contour algorithm. In that formulation, the forces that drive the contour evolution are only computed at the points on the current contour. These values then need to be propagated to the entire domain, as the level set function is defined over the entire domain of the image. This so-called velocity field extension process is computationally expensive. We thus proposed a faster, alternative method: a multiscale active contour (MSAC) transformation framework that uses a geometric embedding of the function but does not involve the distance transform to build the level set function [12]. The MSAC transform combines the flexibility and accuracy of the active contours with the adaptivity and efficiency of multiscale transforms. This is done by casting the energy functionals or forces that drive the active contour evolution as convolutions, permitting an FFT-based implementation of the algorithm.

In that algorithm, the contour convergence is facilitated by, but does not critically depend on, a good initial contour. Further, convergence is very quick, as the contour may advance by more than a few pixels in each iteration. Unfortunately, this increased rate of convergence comes at the cost of the loss of topology preservation. Indeed, the topology preserving criterion used in TPSTACS is based on the topological number of a point in the level set function [13], defined in a $3 \times 3$ neighborhood around the point. When, as in the MSAC algorithm, the contour jumps in large increments in a single iteration, such local information is of little use. Instead, what is needed is an algorithm that retains all the advantages of the MSAC transform in addition to accommodating topology preservation as a force in its framework, the proposed goal in this work.

\section{A NOVEL ACTIVE MASK FRAMEWORK FOR SEGMENTATION}

We propose to segment images of multiple cells using multiple masks, rather than the geometric embedding of the contour in a single scalar-valued function. Although multiple level sets have been proposed before [14], the transform perspective of our segmentation core makes the proposed framework a novel approach.

In this design, we regard the segmentation of an image $f(x)$ as the computation of $M$ masking functions $\left\{\psi_{m}\right\}_{m=1}^{M}$, each having the same domain as $f$ yet taking values in $\{0,1\}$, with $\psi_{1}(x)+\cdots+\psi_{M}(x)=1$, for each $x \in \mathbb{R}^{2}$. That is, each $\psi_{m}(x)$ is a mask (indicator function) of the $m$ th segmented region. The forces that drive the segmentation are based on the properties of the image or on the desired properties of the mask, such as having smooth boundaries, etc. As the forces act on the masks and it is the masks that evolve, we call our method an active mask (AM) algorithm. The segments of the original image are immediately available as the masked components $f_{m}(x)=\psi_{m}(x) f(x)$. Moreover, by combining the different masks, we may obtain a single mask with multiple regions, each of which corresponds to a cell; Fig. 1 depicts the $\psi_{m}$ 's corresponding to an image with three cells, together with their Golgi bodies, represented as one mask. This formalism is well suited to our application, as the volume of the cells can be easily obtained by integrating over the cell areas.

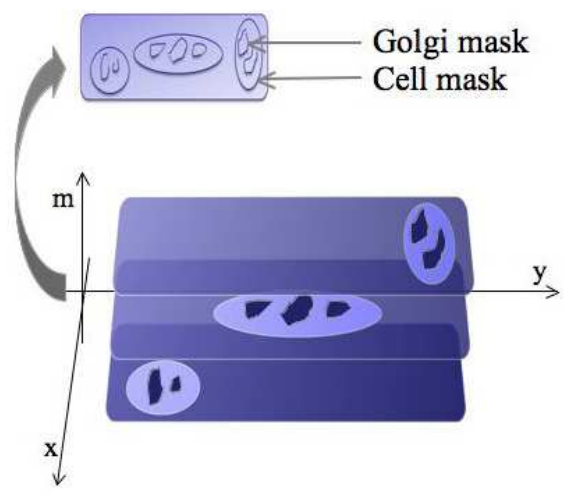

Fig. 1. A schematic representation of the multiple masks, $\psi_{1}, \psi_{2}$ and $\psi_{3}$, corresponding to three cells in an image together with the masks for the Golgi body fragments contained in them. We can combine the information from these separate masks to locate the different cells (and their Golgi body fragments) in an image.

Initialization. We start with a random initialization of $M \gg M_{0}$ masks, where $M_{0}$ is the expected number of cells in the image to be segmented. Each mask $\psi_{m}$ is an indicator function of the pixel value $f(x)$ of the image belonging to the region $m$. As the algorithm progresses, it is likely that some of these indicator functions will converge to zero, that is, empty masks; in this case, we eliminate these masks and reduce the number $M$ accordingly.

Evolution. The active masks evolve based on two different forces. The first is a region-based force $R(x)$. Given the initial configuration, this force first acts on the $M$ masks to coarsely separate the background and foreground regions. As we require only a coarse separation, $R(x)$ is essentially a weighted soft thresholding of a smoothed version of the original image $f$. We use a single mask $\psi_{1}$ to segment the background; the purpose of the remaining masks $\left\{\psi_{m}\right\}_{m=2}^{M}$ is to segment the foreground into approximately $M_{0}$ distinct cells. To be precise, the entire collection of masks $\left\{\psi_{m}\right\}_{m=1}^{M}$ evolves via a local majority voting process, which is skewed according to the region-based force. In particular, our second force, a smoothing "geometric force" is the filtering operation:

$$
F_{g}\left(\psi_{m}(x)\right)=\int_{\mathbb{R}^{2}} \psi_{m}(x) g\left(\frac{x-y}{a}\right) \mathrm{d} y,
$$


where $g$ is a lowpass filter with dilation parameter $a$. We then add the region-based force to $F_{g}\left(\psi_{1}(x)\right)$, which, in essence, grants background pixels a disproportionately large vote to remain in the background. For any pixel $x$, the mask to which the pixel will belong in the next iteration corresponds to the index $m$ which is the maximum of the values $\left\{F_{g}\left(\psi_{1}(x)\right)+\right.$ $\left.R(x), F_{g}\left(\psi_{2}(x)\right), \ldots, F_{g}\left(\psi_{M}(x)\right)\right\}$. In this paradigm, masks "fight" with each other to take over their neighboring region. They stop evolving when the number of points voting on either side of the border is balanced.

Stopping criterion. The geometric force is iterated until the masks converge to a pattern and change no more, that is, when the skewed voting results in no pixels changing masks. This is an advantage as we do not have to choose the number of iterations to run the algorithm as is usually the case with most active contour algorithms.

We display the result by projecting each of the $\psi_{m}$ masks to the space of the same dimension as that of the image to obtain a multihued mask, with each hue representing a different region (see Fig. 3(c)).

\section{CELL-VOLUME COMPUTATION AND GOLGI BODY SEGMENTATION}

We are given a stack of $N$ 2D images with two channels each-one representing the cells and the other, the localization pattern of a Golgi-body protein contained within them. As the Golgi body is an irregularly shaped organelle and very dynamic in nature, we often see multiple fragments of the organelle in the Golgi channel. Thus, segmenting the Golgi channel alone is a difficult task. Further, the cell boundaries are not always well-defined and, especially towards the periphery of the stack, most of the cells are not even discernable. These are some of the problems that complicate the task of segmentation and volume computation.

Golgi body segmentation. We adapt the active mask algorithm to segment the cell images and Golgi body images as follows: First, we initialize the AM algorithm on the middle slice in the stack and segment it to obtain the masks representing various cells in the image. As the cells are fairly distinct in this middle slice, the number of masks in the segmentation output serves as a reliable reference for the number of cells in the stack. After obtaining the active mask segmentation output on this slice, we use the result to initialize the Golgi body segmentation on the corresponding parallel channel image. Then, we propagate these results by using the corresponding masks to initialize the slices preceding and following the middle slices (see Fig. 2). We then repeat the process to segment all of the images in the stack.

Volume computation. Once we obtain the segmentation masks for the cell channel for all slices of the stack, we associate the different cells across the stack by computing the extent of overlap between the masks. We then sum up the areas of the masks that represent the corresponding cell to ob-

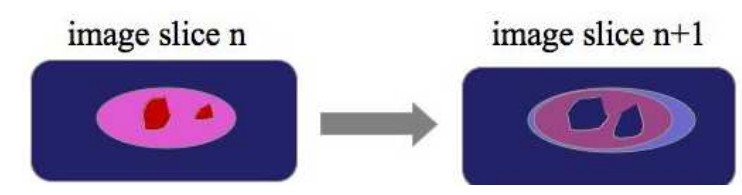

Fig. 2. The segmentation outcome of slice $n$ is used to initialize the masks for segmenting slice $n+1$.

tain the cell volume. We use these cell masks to associate the different Golgi fragments to their respective cells. We then sum up the areas of the corresponding Golgi masks across the stack to obtain the Golgi body volume for each cell.

\section{EXPERIMENTS}

Dataset. Our data consists of 15 fields of images (a z-stack of 2D slices), containing 3-8 cells each. 40 optical sections of size $1024 \times 1344$ pixels, were acquired, starting from the top of the cell to the bottom. The cells are double labeled with Sec13, a soluble membrane-protein and the Golgi marker protein, Giantin, in two parallel channels. Sec13 staining has a diffuse cytoplasmic background, which is used to mark the boundary of the cell. The resolution in $\mathrm{x}, \mathrm{y}$ is $0.05 \mu \mathrm{m}$ whereas the z-resolution is $0.3 \mu \mathrm{m}$. Fig. 3(a) shows an example of the original cell image and Fig. 3(b) the corresponding Golgi body image for an arbitrary slice from the stack.

Results and discussion. We report here the numbers obtained for one of the noisier stacks with a relatively large number of cells. We used an initial number of masks, $M=2^{16}$ to segment the middle slice. We then used the masks of the middle slice to initiate the segmentation of the neighboring slices and repeated the process to the end of the stack. We fixed the resolution of the image to (1/8)th of the original as we obtain a good segmentation performance at this scale, even without further tuning. Fig. 3(c) shows the active mask outcome overlaid with the hand-segmentation result for an arbitrary slice chosen from the stack.

The biologists who visually assessed these results agreed that the AM segmentation is far better and more reliable than the hand segmentation. Thus, they will use the results from the AM algorithm as their reference. To quantify the difference between the AM segmentation and the hand segmentation, we computed the average area similarity $(A S)$ between the masks. According to the literature, an average area similarity of $70 \%$ or greater, indicates a good agreement with the ground truth [15], which is the result of the AM segmentation in this case. We obtain an average $A S=35.19 \pm 23.20$, denoting poor agreement between the hand segmentation with the AM results. This is not surprising as, with hand segmentation, not a single cell has been segmented in slices 32-40. Further, even amongst the more visible slices, the hand segmentation is not reliable. For instance, Fig. 3(c) shows how the cells that exist have not all been chosen by the manual 


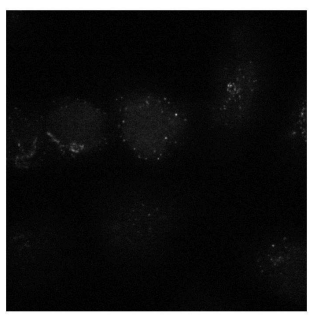

(a)

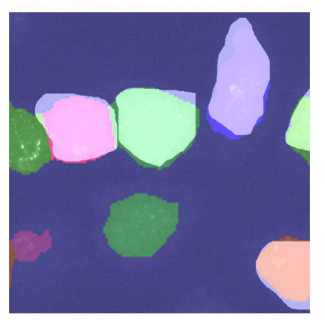

(c)

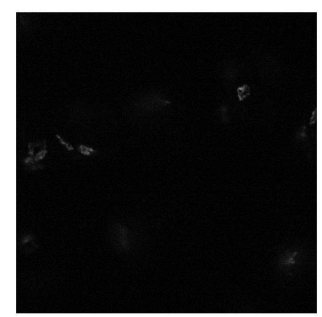

(b)

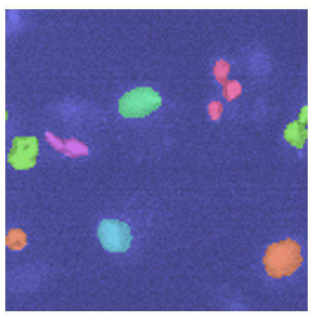

(d)
Fig. 3. Golgi Data and active mask Segmentation results. (a) The original cell image for slice 13. (b) The original Golgi body image for slice 13. (c) The active mask segmentation result: each color corresponds to a different cell, overlaid with the hand-segmentation masks in white. (d) Segmentation of the Golgi fragments with pieces that belong to a cell coded by a different mask.

segmenter and those that have been segmented are not necessarily accurate, a limitation of the human eye in discerning the cells correctly in these images. The forces of the AM algorithm, on the other hand, are designed to extract the features of the cells that aid in their segmentation.

The measure of interest in our application is the volume of the cell and the Golgi body. We computed the total volume of the cells based on the AM masks and compared this with that computed by hand to quantify how much manual processing deviates from the automated method. We found that the difference in the total volume between the AM algorithm and hand segmentation is more than $3 \times 10^{5}$ pixels, that is, more than $28 \%$ of the total number of pixels, showing again the limitation of manual segmentation.

Thus, we cease to compare the performance of the AM algorithm with the hand-segmented masks as such a comparison is not a meaningful one.

We coded the AM algorithm using Matlab ${ }^{\circledR}$ Student Version R2007a. The entire process of segmenting the cells and Golgi bodies and computing the volume for a stack of $40 \mathrm{im}-$ ages takes 5.12 minutes on a $1.86 \mathrm{GHz}$ Core 2 Duo Processor. Thus, the AM algorithm is not only accurate but also efficient and can be used for high-throughput analysis.

\section{SUMMARY}

We have presented a novel active mask segmentation algorithm that combines the advantages of flexibility and accuracy of active contours as well as the efficiency and adaptivity of multiscale transforms, in addition to elegantly incorporating topology preservation as a force. We have demonstrated why this algorithm is more viable than manual segmentation for a quantitative and large-scale study of the properties of Golgi body proteins. We have also shown that the formulation of this algorithm makes it easy to segment and compute the volume of the Golgi body, which has multiple fragments within a cell in a $2 \mathrm{D}$ image.

Acknowledgements. We thank Jia-Shu Chen and Stephen Lin for their preliminary experiments on using z-stack information to initialize the segmentation of peripheral slices in a stack of cell images.

\section{REFERENCES}

[1] J. G. Donaldson and J. Lippincott-Schwartz, "Sorting and signaling at the Golgi complex," Cell, vol. 101, no. 7, pp. 693-6, 2000.

[2] M. Aridor and L. A. Hannan, "Traffic jam: a compendium of human diseases that affect intracellular transport processes," Traffic, vol. 1, no. 11 , pp. 836-51, 2000.

[3] M. Aridor and L. A. Hannan, "Traffic jams II: an update of diseases of intracellular transport," Traffic, vol. 3, no. 11, pp. 781-90, 2002.

[4] M. A. Puthenveedu and A. D. Linstedt, "Subcompartmentalizing the Golgi apparatus," Curr. Opin. Cell Biol, vol. 17, no. 4, pp. 369-75, 2005.

[5] Y. Guo and A. D. Linstedt, "COPII-Golgi protein interactions regulate COPII coat assembly and Golgi size," Journ. Cell Biol., vol. 174, no. 1, pp. 53-56, 2006.

[6] J. A. Sethian, Level Set Methods and Fast Marching Methods, Cambridge Univ. Press, 1999.

[7] A. Sarti, C. O. Solorzano, S. Lockett, and R. Malladi, "A geometric model for 3-D confocal image analysis," IEEE Trans. Biomed. Eng., vol. 47, no. 12, pp. 1600-1609, 2000.

[8] B. Appleton and H. Talbot, "Globally optimal geodesic active contours," Journ. Math. Imag. Vis., vol. 23, no. 1, pp. 67-86, 2005.

[9] D. R. Padfield, J. Rittscher, T. Sebastian, N. Thomas, and B. Roysam, "Spatio-temporal cell cycle analysis using 3d level set segmentation of unstained nuclei in line scan confocal fluorescence images," in Proc. IEEE Int. Symp. Biomed. Imaging, Apr. 2006, pp. 1036-1039.

[10] L. Coulot, H. Kischner, A. Chebira, J. M. F. Moura, J. Kovačević, E. G. Osuna, and R. F. Murphy, "Topology preserving STACS segmentation of protein subcellular location images," in Proc. IEEE Int. Symp. Biomed. Imaging, Arlington, VA, Apr. 2006, pp. 566-569.

[11] G. Srinivasa, A. Chebira, L. Coulot, H. E. Kirshner, V. S. Oak, J. M. F. Moura, R. F. Murphy, and J. Kovačević, "A flexible active contour algorithm for the segmentation of fluorescence microscope cell images," BMC Bioinformatics, 2007, To submit.

[12] G. Srinivasa, M. C. Fickus, and J. Kovačević, "Multiscale active contour transformations for the segmentation of fluorescence microscope images," in Proc. SPIE Conf. Wavelet Appl. in Signal and Image Proc., San Diego, CA, Aug. 2007.

[13] X. Han, C. Xu, and J. L. Prince, "A topology preserving level set method for geometric deformable models," IEEE Trans. Patt. Anal. and Mach. Intelligence, vol. 25, no. 6, pp. 755-768, Jun. 2003.

[14] L. A. Vese and T. F. Chan, "A multiphase level set framework for image segmentation using the mumford and shah model," Int. Journ. Comp. Vis., vol. 50, no. 3, pp. 271-293, 2002.

[15] C. Pluempitiwiriyawej, J. M. F. Moura, Y.-J. L. Wu, and C. Ho, "STACS: A new active contour scheme for cardiac MR image segmentation," IEEE Trans. Med. Imag., vol. 24, no. 5, pp. 593-603, May 2005. 